\title{
2021 Reviewer Acknowledgment
}

\section{Editorial Office of Aging Pathobiology and Therapeutics}

Aging Pathobiology and Therapeutics would like to express our appreciation and recognition to the peer reviewers from all over the world in the year of 2021. Their rigorous scholarship and selfless dedication ensured the high quality and standards of the Journal. And it was a great honor for the editors and authors to benefit from their hard work. They include:

\section{Adam Salmon}

University of Texas Health Science Center at San Antonio, Barshop Institute for Longevity and Aging Studies, San Antonio, TX, USA.

\section{Amrita Basu}

Department of Medicine, University of Texas Health San Antonio, 7703 Floyd Curl Dr, MC 7882, San Antonio, TX 78229, USA (K. Sharma). SVR BioScience Research Services, Salboni 721132, PaschimMidnapore, West Bengal, India.

\section{Ardalan Shariat}

Sports Medicine Research Center, Neuroscience Institute, Tehran University of Medical Sciences, Tehran, Iran.

\section{Axel Kowald}

UK National Innovation Centre for Ageing, The Catalyst, 3 Science Square, Newcastle University, Newcastle upon Tyne NE4 5TG, UK; Rostock University Medical Center, Institute for Biostatistics and Informatics in Medicine and Aging Research (IBIMA), Rostock, Germany.

\section{Beibei Zhang}

Institute of Biomedicine, Yunnan University, Kunming, Yunnan, China.

\section{Brian D. Adams}

Baystate Medical Center, 759 Chestnut Street, Springfield, USA.

\section{Christina Sigurdson}

Departments of Pathology and Medicine, University of California, San Diego, La Jolla, CA, USA.

\section{Denny Liggitt}

Department of Comparative Medicine, School of Medicine, University of Washington, Seattle, WA, USA.

\section{Donghui Liu}

Department of Geriatrics, Guangzhou First People's Hospital, Guangdong, China. 


\section{Guanjun Yang}

Associate research fellow School of Marine Sciences, Ningbo University, Zhejiang, China.

\section{Han-Mou Tsai}

Division of Hematology/Oncology, State University of New York Downstate Medical Center, 450 Clarkson Ave, Brooklyn, NY, USA.

\section{Hanyu Liang}

Department of Medicine-Diabetes Division, University of Texas Health Science Center at San Antonio, San Antonio, TX, USA.

\section{Iman Fatemi}

Research Center of Tropical and Infectious Diseases, Kerman University of Medical Sciences, Kerman, Iran.

\section{Ioana Cezara Caba}

Center for Advanced Research and Development in Experimental Medicine (CEMEX), "Grigore T. Popa" University of Medicine and Pharmacy, 16 Universității Street, Iaşi, Romania.

\section{Jing Luo}

Institute of Reproductive Health, Tongji Medical College, Hubei province, China.

\section{Jing Yang}

Wuhan Institute of Biological Products, Hubei, China.

\section{José López Castro}

Department of Internal Medicine, Monforte Public Hospital, Lugo, Spain.

\section{Jose Vilches-Moure}

Department of Comparative Medicine, School of Medicine, Stanford University, Palo Alto, CA, USA.

\section{Juan Li}

Department of Respiratory Medicine, The First Affiliated Hospital of Zhengzhou University, 450052, Zhengzhou, China.

\section{Jun Lin}

Department of Orthopedics, First Affiliated Hospital of Soochow University, Jiangsu, China.

\section{Kaoru Tominaga}

Division of Functional Biochemistry, Department of Biochemistry, Jichi Medical University, 3311-1 Yakushiji, Shimotsuke, Tochigi 329-0498, Japan.

\section{Ketan Vagholkar}

Department of Surgery, Dr. D. Y. Patil Medical College, Navi Mumbai, India.

\section{Liang Wang}

Department of Hematology, Beijing Tongren Hospital, Capital Medical University, Beijing, China.

\section{Lijian Zhang}

Department of Neurosurgery, Affiliated Hospital of Hebei University, Hebei, China. 


\section{Martin Darvas}

Departments of Pathology, University of Washington, Seattle, WA, USA.

\section{Michal Masternak}

Division of Metabolic and Cardiovascular Sciences, Burnett School of Biomedical Sciences, College of Medicine, University of Central Florida, Orlando, FL, USA.

\section{Min Zhu}

Department of Neurology, Shandong Provincial Hospital, Shandong, China.

\section{Rafael Alvarez Gonzalez}

Universidad Autónoma de Guadalajara, Decanato de Ciencias de la Salud, Guadalajara, Jalisco, Mexico.

\section{Robert T Means}

Department of Internal Medicine, James H. Quillen College of Medicine, East Tennessee State University, Johnson City, Tennessee, USA.

\section{Shyh Poh Teo}

Department of Internal Medicine, Raja Isteri Pengiran Anak Saleha (RIPAS) Hospital, Jalan Putera Al-Muhtadee Billah, Bandar Seri Begawan, Brunei Darussalam.

\section{Tsuyoshi Fukushima}

Section of Oncopathology and Regenerative Biology, Department of Pathology, Faculty of Medicine, University of Miyazaki, Miyazaki, Japan.

\section{Warren Ladiges}

Geropathology Research Network, Department of Comparative Medicine, School of Medicine, University of Washington, Seattle, WA, USA.

\section{Wei Gao}

Department of Geriatrics, Sir Run Run Hospital, Nanjing Medical University, Jiangsu, China.

\section{Wei Quan}

Xi'an Mental Health Center, East Section of Hangtian Avenue, Shaanxi, China.

\section{Xin Xu}

Department of Geriatrics, Hematology \& Oncology Ward, Guangzhou First People's Hospital, School of Medicine, South China University of Technology, Guangzhou, China.

\section{Yong Zhang}

Department of Transplantation Immunology/Institute of Systematic Genetics, West China Hospital, Sichuan University, Sichuan, China.

\section{Yuji Ikeno}

Department of Pathology, The Barshop Institute for Longevity and Aging Studies, The University of Texas Health Science Center at San Antonio, San Antonio, TX, USA.

\section{Zhenhan Deng}


Department of Sports Medicine, First Affiliated Hospital of Shenzhen University, Shenzhen Second People's Hospital, Guangdong, China.

\section{Zipei Jiang}

Department of Ophthalmology, The First Affiliated Hospital of Wenzhou Medical University, Zhejiang, China. 